\title{
Frequency analysis of electroencephalogram recorded from a bottlenose dolphin (Tursiops truncatus) with a novel method during transportation by truck
}

\author{
Fuyuko Hashio $\cdot$ Shinichi Tamura $\cdot$ Yasunori Okada $\cdot$ \\ Shigeru Morimoto $\cdot$ Mitsuaki Ohta $\cdot$ Naoyuki Uchida
}

Received: 4 September 2009/Accepted: 1 April 2010/Published online: 29 April 2010

(C) The Author(s) 2010. This article is published with open access at Springerlink.com

\begin{abstract}
In order to obtain information regarding the correlation between an electroencephalogram (EEG) and the state of a dolphin, we developed a noninvasive recording method of EEG of a bottlenose dolphin (Tursiops truncatus) and an extraction method of true-EEG (EEG) from recorded-EEG (R-EEG) based on a human EEG recording method, and then carried out frequency analysis during transportation by truck. The frequency detected in the EEG of dolphin during apparent awakening was divided conveniently into three bands $(5-15,15-25$, and $25-40 \mathrm{~Hz}$ ) based on spectrum profiles. Analyses of the relationship between power ratio and movement of the
\end{abstract}

F. Hashio $(\bowtie) \cdot N$. Uchida

Department of Marine Science and Resources,

College of Bioresource Science, Nihon University,

1866 Kameino, Fujisawa 252-8510, Japan

e-mail: fuyuko-h@tokai.or.jp

S. Tamura

Sensor Development Division, Yazaki Meter Co. Ltd.,

Shizuoka 427-0024, Japan

e-mail: tamura.s1@smd.yzk.co.jp

Y. Okada

Medical System Education Center Inc., Tokyo 113-0034, Japan

e-mail: okada@kenn.co.jp

\section{S. Morimoto}

Faculty of Education and Human Sciences,

Yokohama National University, Kanagawa 240-8501, Japan

e-mail: mrmt@edhs.ynu.ac.jp

\section{Ohta}

Department of Animal Science and Biotechnology,

Azabu University Graduate School of Veterinary Medicine,

Kanagawa 229-8501, Japan

e-mail: mohta@azabu-u.ac.jp dolphin revealed that the power ratio of dolphin in a situation when it was being quiet was evenly distributed among the three bands. These results suggested that the EEG of a dolphin could be detected accurately by this method, and that the frequency analysis of the detected EEG seemed to provide useful information for understanding the central nerve activity of these animals.

Keywords Brain - EEG - Fast Fourier Transform · Tursiops truncatus

\section{Introduction}

The bottlenose dolphin (Tursiops truncatus) is a marine mammal positioned at the top of the food chain. The dolphins are organized into highly structured communities known as groups, in which the sex and age of members in the community are balanced to fulfill the functions of the group, such as the feeding system [1]. A group feeds using a process, the chasing method, in which the roles of individual members in the community are systematically connected. The method is adapted to suit the particular situation, including the climate and the kind and amount of the catch [1]. The ability of bottlenose dolphins to carry out such highly organized behaviors as the chasing method seems to be derived from their advanced communication skills, which are made possible by a social ability that rivals that of primates [1]. These brain functions are controlled by the electrical activity of neurons in the cerebral cortex, and changes in this activity can be detected by an electroencephalogram (EEG) or electrocorticogram (ECoG).

Much information is now available about the relationship between a physiological state and EEG in primates, 
including humans. There are a lot of reports of EEG in humans by means of electrodes sitting on the scalp surface and analyzed with regard to the effect of seizure disorder [2] on active parts of the brain, frequency, amplitude and rhythm, changes in overall active level of the brain according to sleep and awakening state [3] and consciousness disorder [4], and the effect of a morbid state [5] on brain function. The results have revealed significant effects of the behavioral state on specific frequencies in the EEG, such as $\alpha$-wave $(8-13 \mathrm{~Hz}$ ).

On the other hand, there have been few reports about the brain waves of whales, which have been recorded by ECoG using electrodes implanted surgically in the cerebral cortex of whales such as bottlenose dolphin [6, 7], white whale (Delphinapterus leucas) [8], boto (Inis geoffrensis) [9], and harbor porpoise (Phocoena phocoena) [10]. These studies only examined the relationship between the ECoG amplitude and sleeping of dolphins, but were not frequency analyses which can be expected to provide more useful information.

Murayama et al. [11] recorded the EEG of a bottlenose dolphin by a noninvasive recording method by positioning electrodes on the scalp surface of the dolphin and analyzed the frequency at less than $1 \mathrm{~Hz}$. However, the dolphin had been kept on shore out of an aquarium, which was widely different from the natural conditions.

The continuous recordings of whale brain waves during various movements with a noninvasive recording method similar to the EEG recording method in humans, and the analysis of frequencies in the brain waves, are expected to provide useful information to elucidate not only the correlation between brain waves and the changes of surroundings or movement but also the central nerve activity of whales.

From this point of view, we developed a noninvasive and waterproof recording method of EEG for a bottlenose dolphin based on a human EEG recording method. Further, we discovered a method of frequency analysis of dolphin EEG. We used this method to record the EEG of a dolphin being transported by truck, and then analyzed the detected frequencies by means of power ratio to obtain the frequency information regarding the correlation between the EEG and the state of the dolphin in appearance.

\section{Material and methods}

\section{Material}

The female bottlenose dolphin used in these analyses had been captured offshore from Taiji in the Wakayama prefecture of Japan on 3 December 2002. The dolphin was $216 \mathrm{~cm}$ in body length. The animal was subsequently reared in the World Dolphin Resort Aquarium in Taiji from
5 December 2002 to 26 February 2004, and grew to $258 \mathrm{~cm}$ in body length within this rearing period. Since 27 February 2004, this animal has been housed in the dolphin facility at Azabu University, located in Murotomisaki Muroto, Kochi prefecture. The estimated age of this female dolphin at the time of the recording was 4 years old from the body length [12]. The dolphin was judged to be healthy from the normal growth rate based on an increase of $42 \mathrm{~cm}$ in body length for 1 year and 3 months [12], and judgment by the veterinarian in the World Dolphin Resort Aquarium according to blood examinations. The actual experiment and treatment were executed according to the animal experiment guidelines of Azabu University.

\section{Experimental procedures}

The transportation of the dolphin from the World Dolphin Resort Aquarium to Azabu University was conducted using a wet-sling method [13], and the real recorded EEGs (R-EEGs) during the transportation were examined on the truck, stopping the engine to avoid the artifacts originated from the movement of the engine. Details of the transportation and R-EEG recording cycles are shown in Table 1. Briefly, the dolphin was laid on a stretcher and put in a container filled with seawater, which was maintained at $100 \mathrm{~mm}$ below blowhole level to prevent the blowhole from being submerged. Some body parts, including the head, melon, dorsal fin and a part of the caudal fin were regularly sprayed with seawater. The behavior of dolphin under recording of R-EEG was monitored by recording on a video with a DCR-PC video camera (Sony, Tokyo, Japan). The degree of movement was expressed as times of head movement and emitting sonant per period of the video recording (times/s), which were calculated from the pictures played back from the video.

The silver plate electrodes coated with $\mathrm{AgCl}$ prior to use in the EEG recordings were covered with a vinyl chloride suction cup in order to attach them to the wet body surface of the animal (Fig. 1a). The suction cup also served to waterproof the electrodes. Each lead line was twisted together to form a single line to prevent electromagnetic induction. The electrodes were then placed on the scalp surface of the dolphin in accordance with the referential derivation method [14]. The placements of the electrodes were designed to enable the most efficient recording of an electric inductance (electrogram) from the dolphin in the container. To detect R-EEG, we set electrodes close to the brain (recording positions $\mathrm{h}-\mathrm{k}$ shown in Fig. 1b). The positions $\mathrm{a}, \mathrm{b}, \mathrm{d}$, and e were selected for detecting the electrogram originated from the breath, also $\mathrm{c}$ and $\mathrm{f}$ were for eye movement in order to compare the electrogram profile with the R-EEG. An earth and reference electrodes were placed in front of the dorsal fin resulting from an 


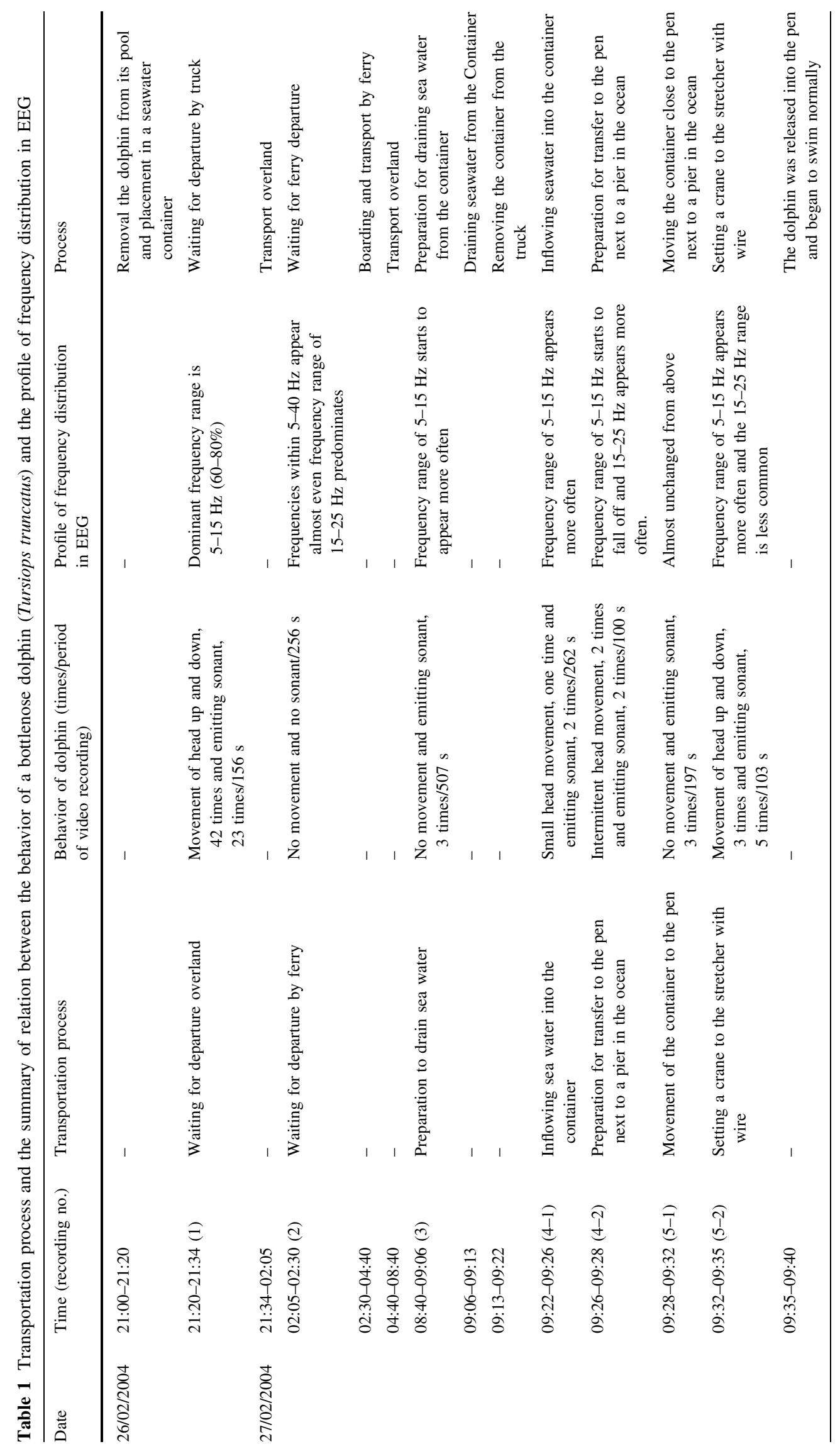



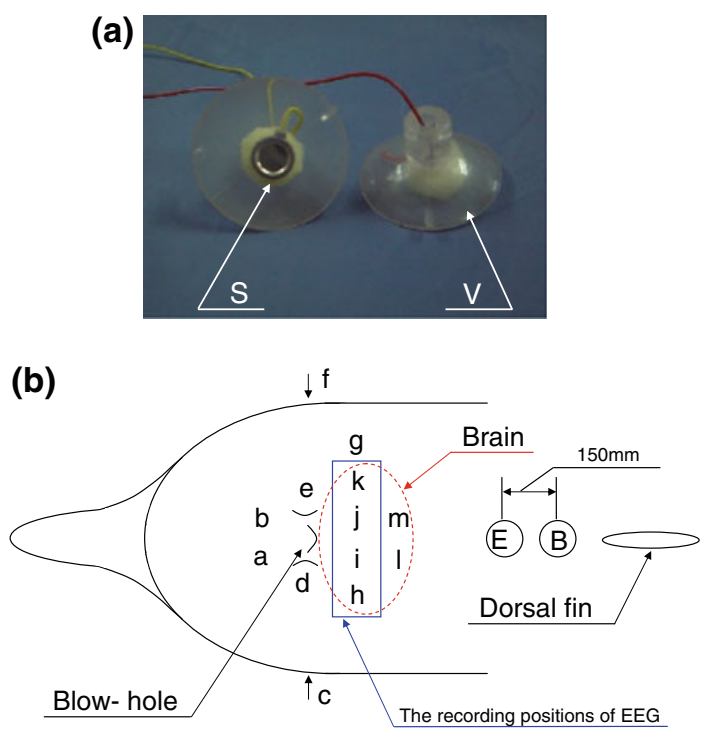

Fig. 1 Silver plate electrode covered with vinyl chloride suction-cup (a) and recording positions (b). Recording positions $c$ and $f$ are above the eyeballs of the dolphin. $S$ Electrode, $V$ vinyl chloride suction-cup, $E$ body earth position, $B$ base electrode position

inspection of the place which would reduce the influence of body and muscle movements on the recording of R-EEG to minimum (Fig. 1).

The R-EEG was transmitted and recorded with an MT-11 telemetry system (NEC Medical System, Tokyo, Japan) as analog data. These data were then digitized with a sampling frequency of $1 \mathrm{kHz}$ using an RA1300 device (NEC Sanei, Tokyo, Japan).

The R-EEG included electrocardiograms (ECGs) and other artifacts. We removed the ECGs from the R-EEG by hand. The ECG signals including p-wave to t-wave indicated in Fig. 2a were removed from the R-EEG and both ends were connected as shown in Fig. $2 b$, and then the ECG-removed R-EEG was applied to the band-pass filter (BPF, Butterworth filter type, 5-40 Hz).

The subsequent frequency analyses were performed using medical frequency analysis software, BIMTUS 2 frequency analyzer (Kissei Comtec, Nagano, Japan). Using the BIMTUS 2 device, we performed a Fast Fourier Transform (FFT) on the digital data of brain wave converted by the RA1300 instrument. The common conditions of FFT were as follows: window function, hamming, and other conditions (interval, number of FFT points, overlap, spectrum, and frequency resolution), were suitably applied as described in the figure legends. The detected rate of the EEG pulse in specific frequency region was expressed as a power ratio defined as the percentage of the power spectrum of a specific frequency region to the power spectrum of entire frequency obtained by the time series of the FFT using a calculation performed with BIMTUS 2 .

\section{Results}

Typical R-EEGs of dolphin under the situation of being quiet are shown in Fig. 2a; the recording situation and movement indicated in Table 1 as recording No. 2. These R-EEGs were detected with the electrode placed on the scalp surface indicated in Fig. $1 \mathrm{~b}$ as recording point $\mathrm{h}-\mathrm{k}$. The R-EEGs detected at recording point $\mathrm{h}-\mathrm{k}$ closely resembled the profile among the recording situation and movement indicated in Table 1 as recording no. 1-5 (not shown). The R-EEGs were composed of pulses with several to several tens of $\mathrm{Hz}$ and the pulses specific to electrocardiograms (ECGs) that were detected at approximately 1.4-s intervals (Fig. 2a, open ellipses).

Although the R-EEGs included ECGs and other artifacts, it was concluded that the R-EEGs originated from the action potential of the brain, judging from the similarity of the main pulse composition to the human EEG with a pulse of $1-30 \mathrm{~Hz}(\delta, \theta, \alpha$ and $\beta$ waves) [14], the clear difference in pulse composition compared with electrograms detected by electrodes at recording points a-g, 1 and $\mathrm{m}$, which were composed of long wavelength pulses (Fig. 3), and resembling the shapes of the electrogram of the dolphin EEG obtained by Murayama et al. [11]. The R-EEGs also frequently contained an instantaneous $(\sim 2 \mathrm{~s})$ artifact pulses that was synchronized with electromagnetic induction that came from breathing, movement of the head, and the electromyogram (EMG) synchronized with movement of pectoral fin. The results of the FFT analysis (HPF, Butterworth filter type, $3 \mathrm{~Hz}$ ) of these instantaneous artifact pulses revealed that the main frequency of the ECG was less than $50 \mathrm{~Hz}$ (Fig. 4b), which completely overlapped with the main frequency of the R-EEG when quiet (Fig. 4a), the main frequency of the EMG was $60-120 \mathrm{~Hz}$ (Fig. 4c), breathing was less than $5 \mathrm{~Hz}$ (Fig. 4d), and movement of the head was less than $5 \mathrm{~Hz}$ (Fig. 4e). Therefore, in order to obtain true-EEG (EEG), the pulses of ECG were deleted manually from the pulse of R-EEG (Fig. 2a, b), and other artifact pulses were removed by processing with BPF of $5-40 \mathrm{~Hz}$ using BIMTUS 2 from the R-EEGs, and then resulting pulse of EEG (Fig. 2c) was applied to frequency analysis.

The frequency analysis of the EEG by the FFT resulted in a spectrum power defined as the detected rate of the frequency pulse. The spectrum power of recording No. 2 was distributed from about 5 to $40 \mathrm{~Hz}$, as shown in Fig. 2d. We tentatively divided the spectrum power into three bands (5-15, 15-25 and 25-40 Hz) on the basis of the following two results. First, the spectrum power was distributed as three frequency bands, with a maximum level of approximately 5, 20, and $35 \mathrm{~Hz}$, and minimum levels at approximately 15, 25, and $40 \mathrm{~Hz}$, respectively (Fig. 5a-c). Second, a spectrum power profile of these frequency bands closely 


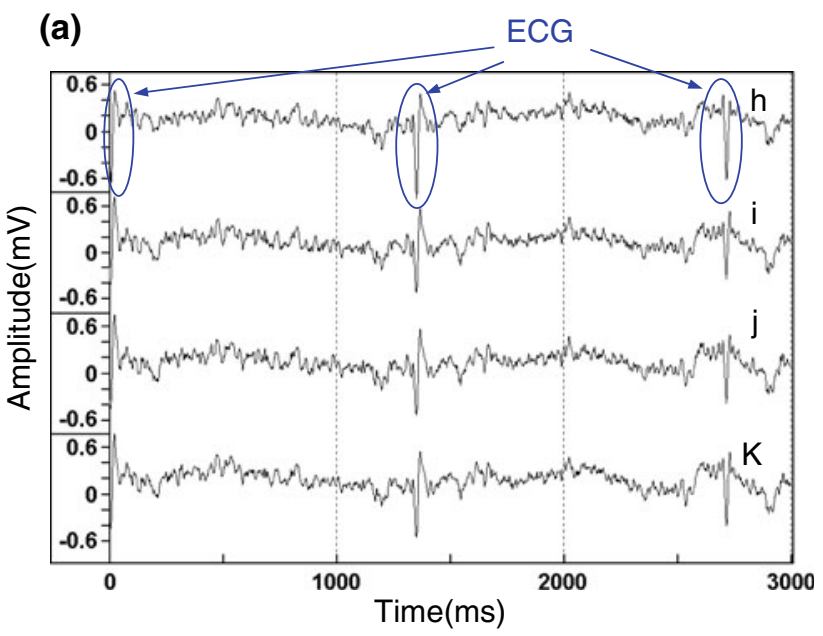

(b)

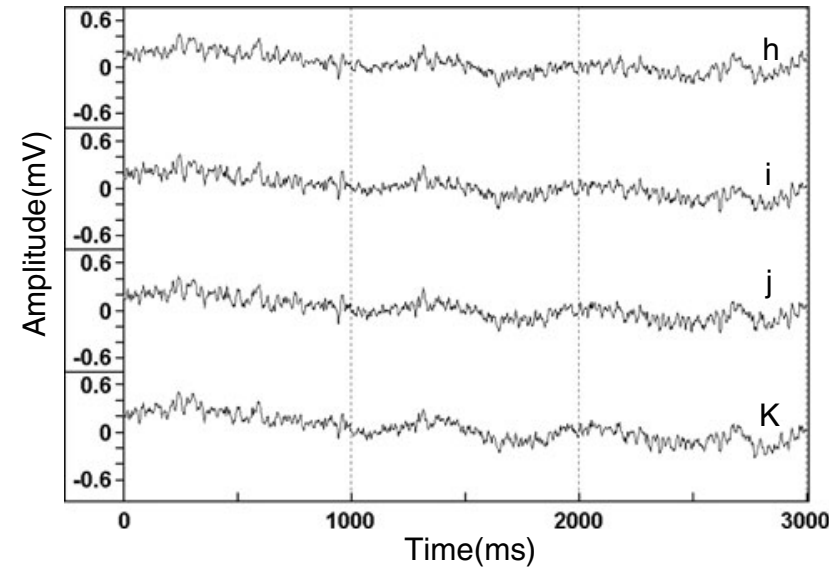

Fig. 2 a Typical pulse profile of recorded-EEG (R-EEG) of dolphin when quiet (recording No. 2 in Table 1); b pulse profile of R-EEG that was a manually removed pulse of ECG from pulse of R-EEG in (a); c pulse of true-EEG (EEG) in which other artifact pulses were

reflected the movement of dolphin, that is, the dolphin of recording No. 3 under low level reaction to stimulation (no movement and emission of a sonant) showed increasing spectrum power of 5-15 Hz, whereas the $15-25 \mathrm{~Hz}$ spectrum power slightly increased and lowered the maximum frequency, and the $25-40 \mathrm{~Hz}$ band maintained the profile (Fig. 5b) compared with that of recording No. 2 under quiet conditions (no movement and no sonant) (Fig. 5a). During recording No. 1, the dolphin reacted strongly to stimulation, moving its head up and down at all times and emitting a sonant, and significantly increased the spectrum power at 5-15 Hz (Fig. 5c) compared with recording No. 2-5.

These results suggested that the spectrum power in the three frequency regions was closely connected with the movement of the dolphin. Therefore, we calculated the time-course of the power ratio of the three bands $(5-15$, (c)

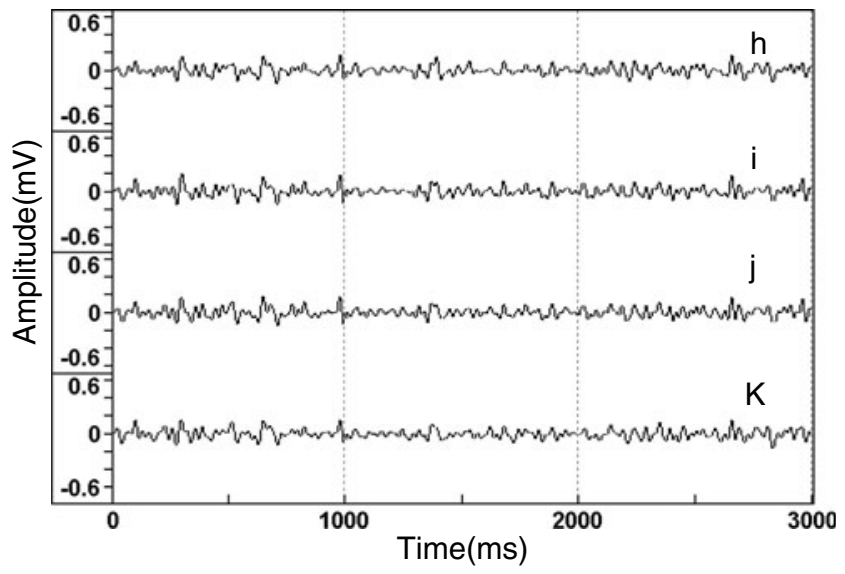

(d)

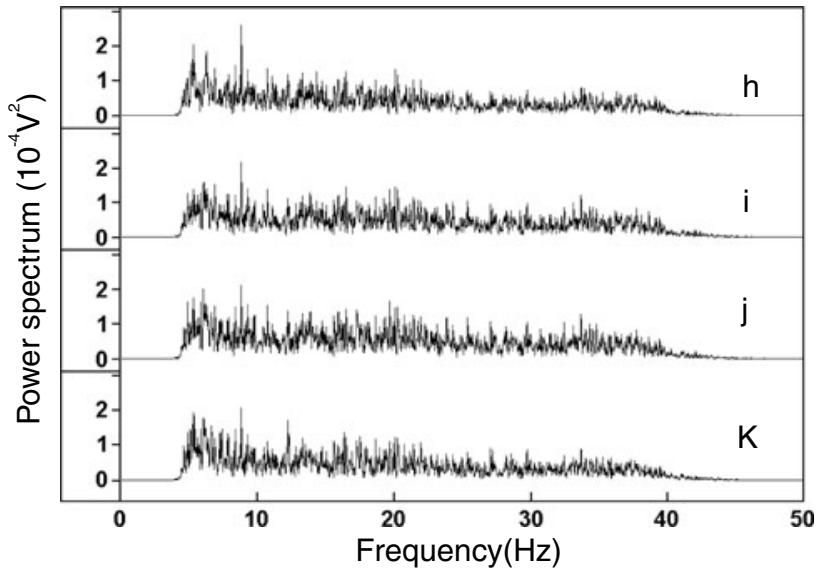

removed from the pulse of ECG-removed R-EEG in (b) by a bandpass filter; $\mathbf{d}$ the power spectrum resulting from frequency analysis of the EEG (c) by Fast Fourier Transform at recording positions $h-k$ in Fig. 1b

15-25, and 25-40 Hz; Fig. 6) from the spectrum power obtained from the EEGs of dolphin under the situation of recording No. 1 5-2 in Table 1. The relationship between the movement of the dolphin and the time-course profile of power ratio is summarized in Table 1.

The power ratio of the dolphin in recording No. 2, which was considered to be when quiet (Table 1), was almost even among the three bands and was maintained at constant levels within the recording period. In recording No. 3, the dolphin showed slight reactions to stimulation, and there was a clear increase in the $5-15 \mathrm{~Hz}$ band compared with the other two frequency regions. Moreover, this did not change during the recording period. During recording No. 1, the dolphin was judged to be indicating a violent reaction to stimulation from the movement, the power ratio of $5-15 \mathrm{~Hz}$ band increased rapidly, while those of the $15-25$ and $25-40 \mathrm{~Hz}$ bands 


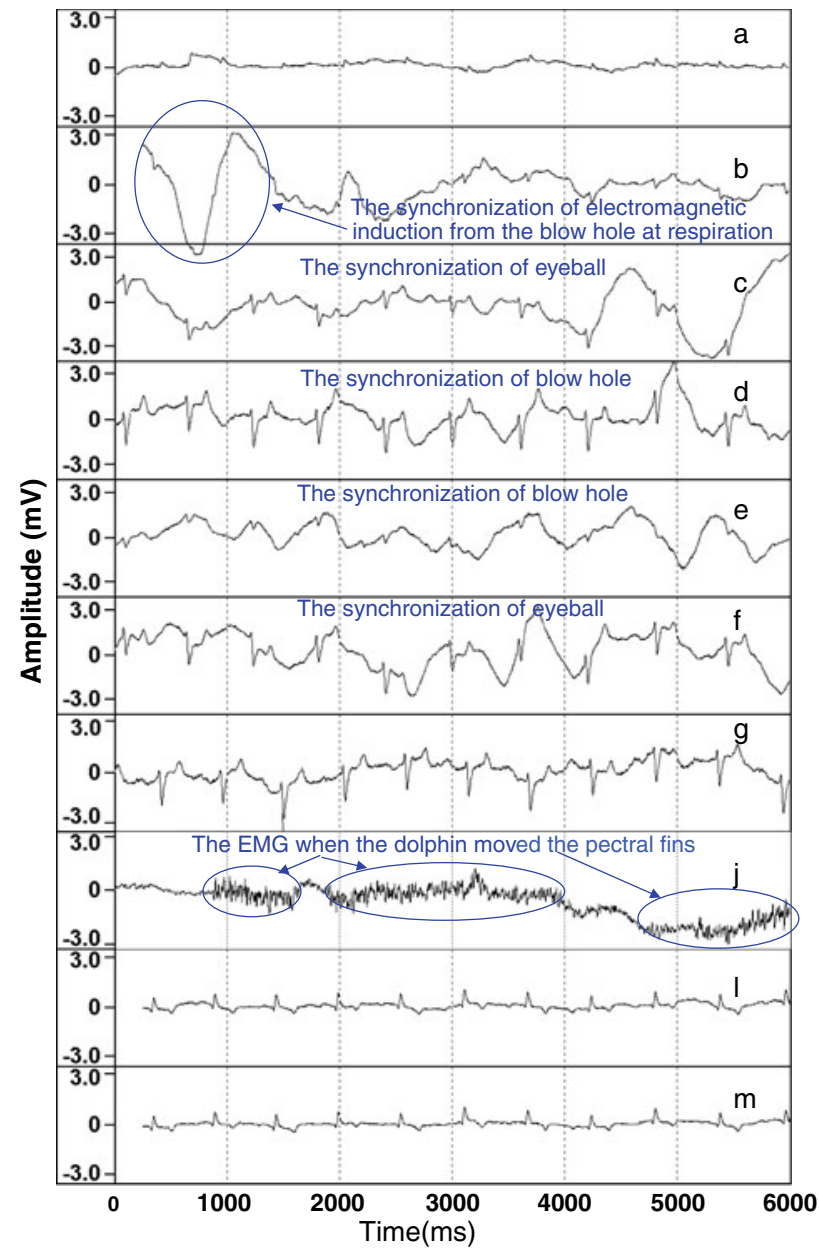

Fig. 3 Typical R-EEG of dolphin when quiet (recording No. 2 in Table 1) recorded at recording position $j$ and electrograms of $a-g, l$, and $m$ in Fig. $1 \mathrm{~b}$

reduced. The dolphin under the situation of recording No. 4 was receiving relatively strong stimulation by an inflow of seawater in the first half of the recording period (recording No. 4-1) and was released gradually from the stimulation in the latter half (recording No. 4-2). During recording No. 4-1, the dolphin displayed small head movements and emitted a sonant, and the power ratio of the $5-15 \mathrm{~Hz}$ band increased in a similar manner to recording No. 3. In recording No. 4-2, the power ratio of the three bands settled at equal values according to a decrease of movement (intermittent head movement and emission of a sonant). In recording No. 5, The dolphin did not move but emitted a sonant during the early stage (No. 5-1), but occasionally moved its head with sonant emission during the later stages (No. 5-2). At first, there were no clear differences among the power ratio of the three bands (No. 5-1), but increases in the power ratio of the $5-15 \mathrm{~Hz}$ band led to clear differences (No. 5-2).

\section{Discussion}

It has been widely reported that bottlenose dolphins have advanced communication skills that might be based on a high intellectual ability, which is similar to that of primates [1]. These brain functions are controlled by the electrical activity of the neurons in the cerebral cortex, and any changes in this activity can be detected as the EEG [14]. In primates such as humans, a relationship between the physiological situation and the frequency of the EEG has been elucidated, and the results have been applied to medical examinations [2-5].

There are, however, two noteworthy differences between the EEG recording conditions for humans and dolphins. One is a strong movement of the dolphins underwater, making it necessary to fix a waterproof electrode on the body surface of dolphin. The other is that artifacts such as ECG are more easily included in R-EEGs of dolphins than humans. These are due to the fact that the electric signal of ECG is conducted from heart to head by volumetric conduction [14]. In humans, the R-EEG is not interfered with by ECG resulting from a remarkable attenuation of ECG by a high conductive resistance owing to distance and isolation between heart and head. In contrast, R-EEG of dolphins is affected by ECG because a small attenuation is caused by the low impedance on the pathway owing to the short distance and lack of narrowing between heart and head, resulting in ECG existing at the frequency region that might be the frequency region of EEG. Therefore, it is necessary to eliminate the ECG from the R-EEG in order to extract the true-EEG. EMG and electromagnetic induction could be canceled by BPF.

There are few studies of brain waves of whales. The majority of them were recorded by ECoG by an invasive recording method in which an electrode was implanted surgically into the cerebral cortex [6-10]. These ECoG analyses on sleeping of whales revealed that the whales were unihemispheric sleepers [6-10], and that sleeping stage of bottlenose dolphins could be classified into three judging from the ECoG amplitude intensity, in which the ECoG amplitude was in inverse proportion to the degree of sleep, and suggested that the only frequencies detected were $4 \mathrm{~Hz}$ or less in sleeping with maximum ECoG amplitude [6, 7]. These studies, however, were not subject to further frequency analysis.

Murayama et al. used a noninvasive recording method to record EEG of a bottlenose dolphin on shore out of an aquarium by positioning electrodes on the scalp surface of the dolphin, but did not eliminate ECG and other artifacts from the recorded-EEG (R-EEG) [11]. In addition, these studies did not include frequency analysis of the R-EEGs. There are also studies that recorded the brain-stem response of whale, but not EEG, during echolocation by 
Fig. 4 The power spectrum profile resulting from FFT analysis on the EEG when the dolphin was quiet (a), on ECG (b), on EMG synchronized with movement of pectoral fin (c), on electrograms of breathing (d) and moving the head (e). The FFT conditions were as follows: interval, $1.024 \mathrm{~ms}$; window function, hamming; overlap, none; spectrum, power and frequency resolution, $0.977 \mathrm{~Hz}$; the number of FFT points, 651 (a), 418 (b), 68 (c), 387 (d), 719 (e) (a)

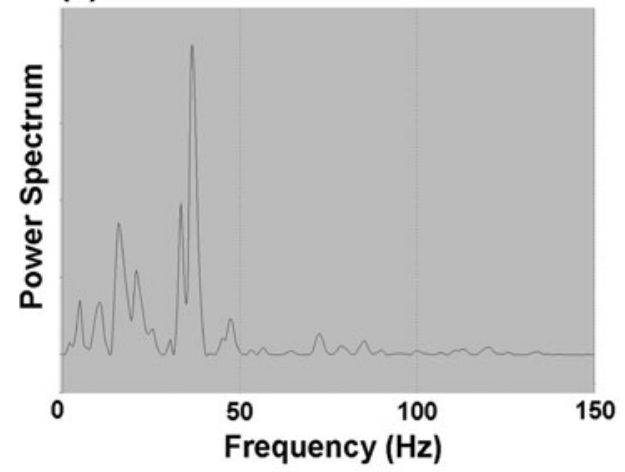

(c)

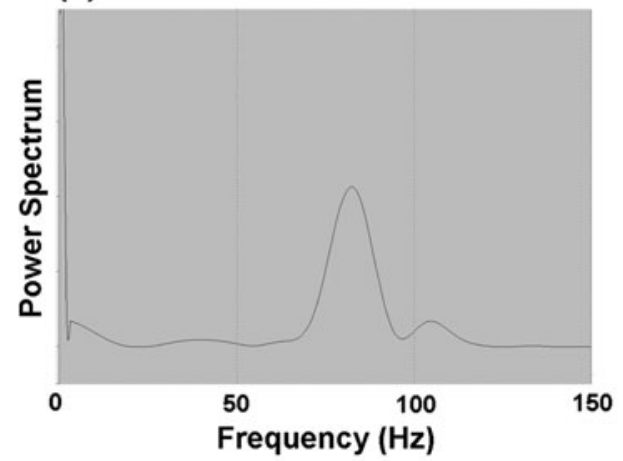

(e)

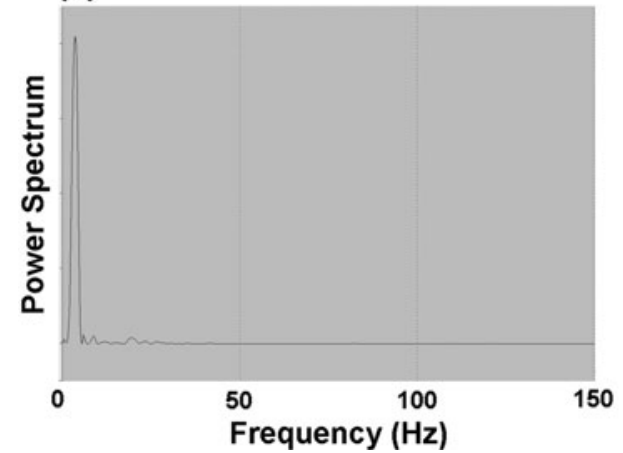

(b)

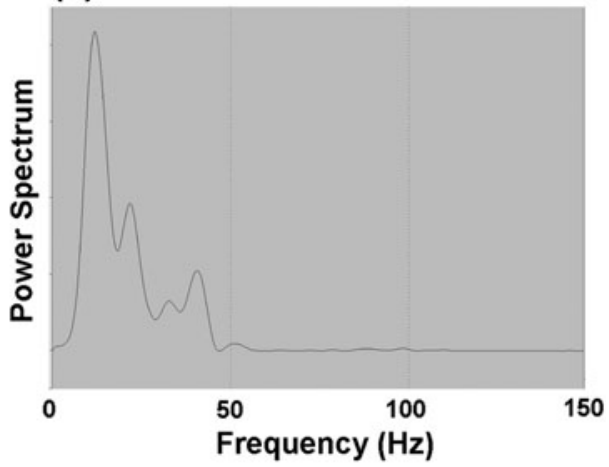

(d)

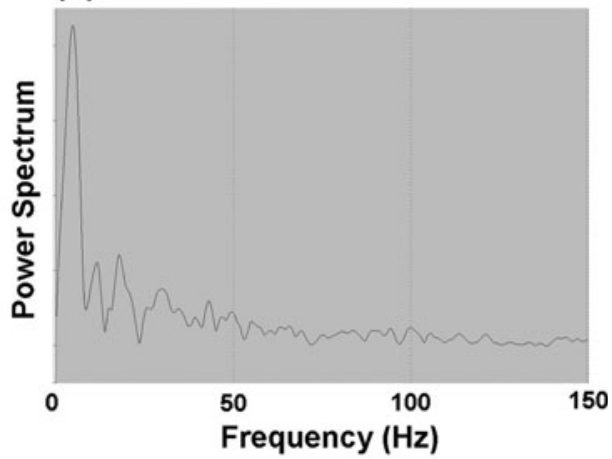

using EEG electrode fixed with suction-cup [15]. To our knowledge, however, no previous study has recorded the EEG of a dolphin using an appropriate analytical method which solved these problems, and then analyzed the frequencies detected therein.

In order to solve the problem of attaching the surface electrodes in this study, we fixed electrodes on the scalp surface by covering them with a vinyl chloride suction-cup, making it possible to firmly fix the electrodes and to waterproof them. This improvement allowed us to continuously record the R-EEG of a dolphin moving vigorously underwater. To solve the problem of artifacts including the R-EEG, we eliminated ECG and other artifacts from the R-EEG through manual manipulation and mechanical filtering, respectively, to extract the EEG. These methods allowed us to analyze the frequency detected in EEG and, to our knowledge, this is the first study that does so in this way.

The EEG of the dolphin during transportation by a truck was detected by this method and the detected frequency was analyzed. The EEG covered in total the region of $5-40 \mathrm{~Hz}$. The spectrum power (continuous change in the spectra with frequency) could be divided into three frequency bands $(5-15,15-25$, and $25-40 \mathrm{~Hz})$. These three dolphin frequency bands roughly correspond to the frequency bands of human EEG as follows: $5-15 \mathrm{~Hz}$ corresponds to the $\theta$-wave $(4-7 \mathrm{~Hz})$ and $\alpha$-wave $(8-13 \mathrm{~Hz}$ ); $15-25 \mathrm{~Hz}$ corresponds to the $\beta$-wave $(14-30 \mathrm{~Hz})$; and $25-40 \mathrm{~Hz}$ corresponds to a part of the $\gamma$-wave $(30-64 \mathrm{~Hz})$. The intensity of the three bands was found to be closely 


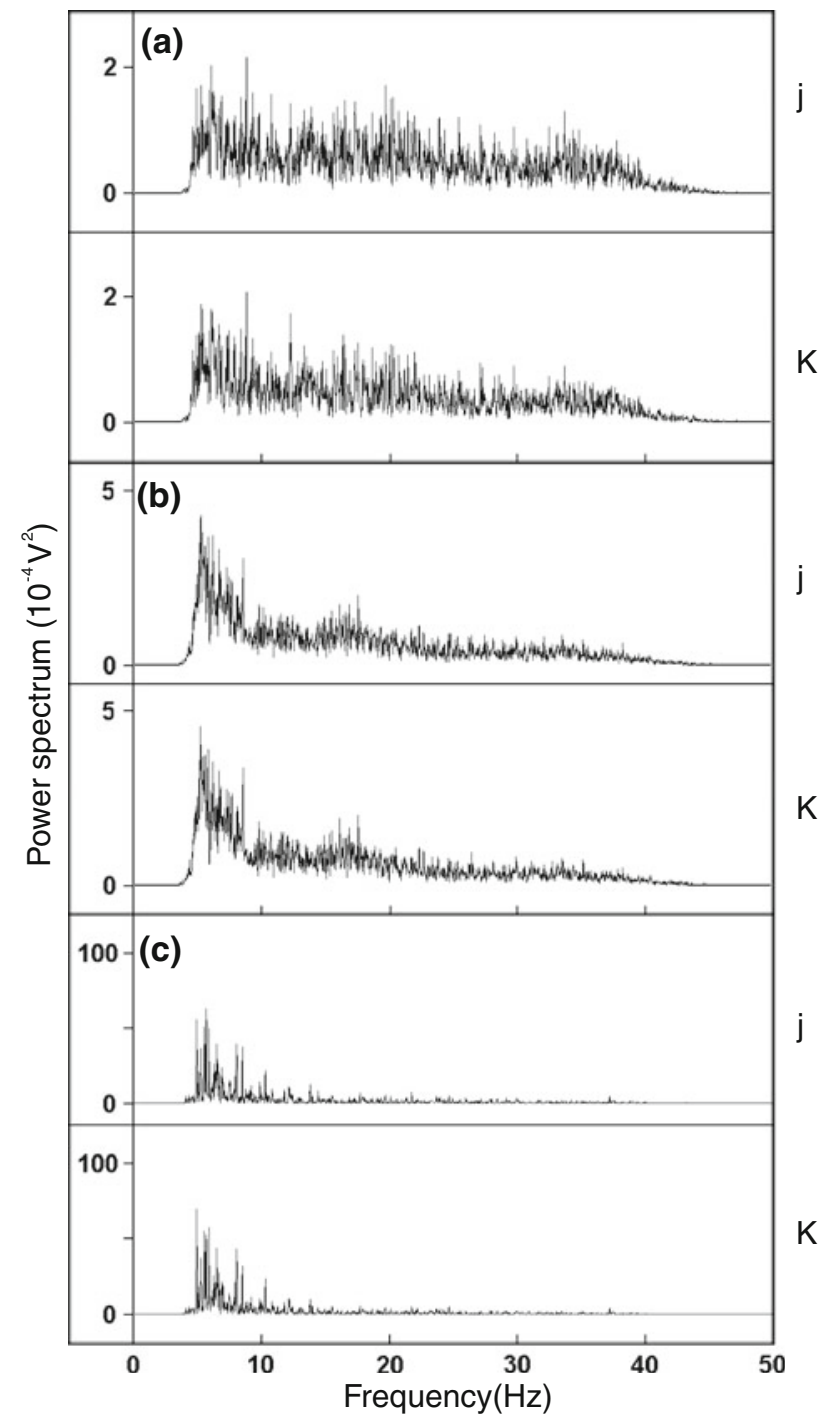

Fig. 5 The spectrum power profile resulting from FFT analysis on the EEG of recording No. 2 (a), No. 3 (b), and No. 1 (c) at recording position $\mathrm{j}$ in Fig. 1b. The FFT conditions were as follows: interval, $1.024 \mathrm{~ms}$; the number of FFT point, 32768; window function, hamming; overlap, 1/2; spectrum, power and frequency resolution, $0.031 \mathrm{~Hz}$

related to the degree of reaction to exterior stimulation. The $5-15 \mathrm{~Hz}$ bands, in particular, the lower part of which roughly corresponds to the $\theta$-wave in human, increased with increasing reactions to exterior stimulation, but the other frequency bands did not clearly change in response to movement. This response is in contrast to human EEG, in which $\beta$-waves are dominant when the subject is awake and are highly responsive to exterior stimulation, $\alpha$-waves are dominant under relaxed conditions, and $\theta$-waves are dominant during the subject is dozing [16].

These results suggest that the frequency of dolphin EEG shows similar profiles to that of humans, but the physiological significance of these frequency bands differs significantly from that in humans.

We propose from our present data that our novel, improved method is a useful procedure for recording the EEG of dolphins and then performing frequency analysis, and that the EEG could be successfully extracted from the R-EEG by this method because we could cancel the expected artifacts such as ECG, EMG, and electromagnetic induction. It is commonly accepted that the frequency of EMG recording on the outer layer of human skin consists of the bandwidth of $50 \mathrm{~Hz}$ or less [17, 18]. In this present study, however, this low frequency band was not recognized in EMG originated from movement of the dolphin, which was recorded at the R-EEG recording position $\mathrm{j}$ (Fig. 4c). The EMG of the moving dolphin was mainly generated from the muscles in the vicinity of mandible and the pectoral fin, which were far from the recording position (about $80 \mathrm{~cm}$ in this study) in comparison with recording of human EMG. The EMG was transmitted to the electrode by the volume conduction, and the amplitude of the EMG was attenuated during the long-distance transmission resulting in an undetectable low frequency band in the EMG at the R-EEG recording position [19]. Furthermore, an instantaneous $(\sim 2-\mathrm{s})$ pulse synchronized with the movement of the dolphin seemed to be not effected on the R-EEG recording for a long time (100-507 s). These considerations lead to our conclusion that the influence of EMG on the EEG may not be able to be rejected completely, but is negligible.

These results also demonstrate that variations in the power ratio of the three bands are closely related to the movement of the dolphin, and increases in the power ratio of $5-15 \mathrm{~Hz}$ band show a particularly strong correlation with increased reactions to stimulation.

It is, however, necessary to perform this method many times because the frequency analysis in R-EEG involves several analytical steps such as ECG removal, filtering, and time-series analysis. Recently, digital data processing with a microcomputer has made it possible to construct specialized systems to analyze the R-EEG data, resulting in easy and immediate analysis of the dolphin EEG.

We propose that accumulating the available data on the relationship between dolphin movement and EEG frequency by using the specialized methods for brain wave recording and EEG analysis outlined herein may make it possible to infer the presence of nervous system disease or brain injury in the dolphin as well as in the human from a noninvasively recorded EEG, and that the noninvasive EEG analysis could be used to elucidate valuable information that will assist with health control in these sea animals. 
Fig. 6 Change of power ratio with recording time. Power ratio are calculated from the power spectrum obtained from FFT analyses of the EEG of dolphin with the movement listed in Table 1 as recording No. 1 (1), No. 2 (2), No. 3 (3), No. 4-1 (4-1), No. 4-2 (4-2), No. 5-1 (5-1), and No. 5-2 (5-2). The FFT conditions were as follows: interval, $2.048 \mathrm{~s}$; window function, hamming; overlap, $1 / 2$; spectrum, power and frequency resolution, $0.488 \mathrm{~Hz}$; the number of FFT points, 76 (1), 140 (2), 251 (3), $112(4-1)$, $100(4-2), 96(5-1), 65(5-2)$

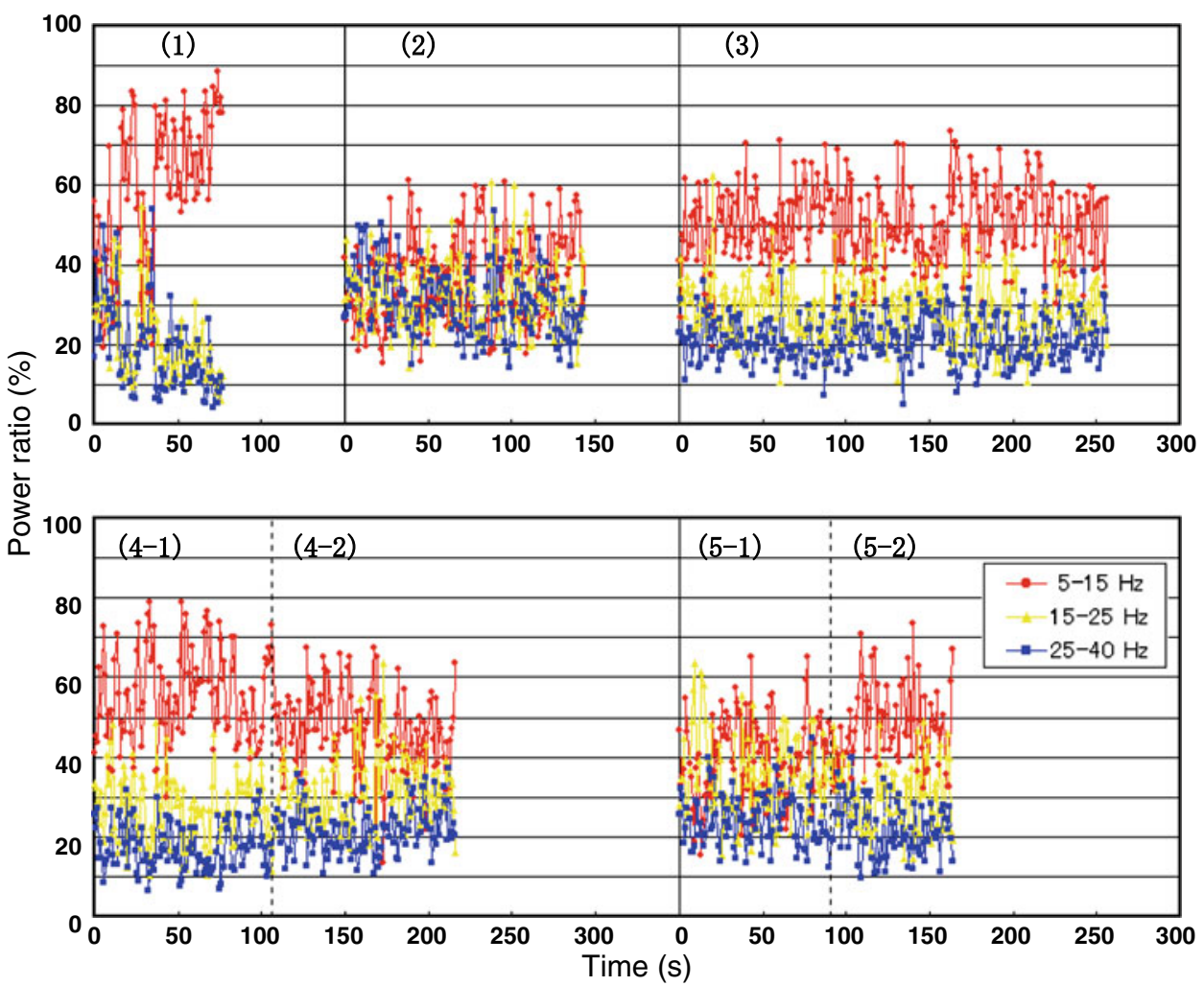

Acknowledgments We would like to thank Mr. Kenji Maejima, Mr. Kaoru Koyama and Mr. Noriyasu Suzuki, Izu-Mito Sea Paradise, Shizuoka, Japan for their kind advice, and Mr. Yoichi Kurihara and Ms. Kana Suga, World Dolphin Resort, Wakayama, Japan, for their help in our experiment.

Open Access This article is distributed under the terms of the Creative Commons Attribution Noncommercial License which permits any noncommercial use, distribution, and reproduction in any medium, provided the original author(s) and source are credited.

\section{References}

1. Pryor K, Norris KS (1998) Dolphin societies. University of California Press, California

2. Whitsett SF, Lubar JF, Holder GS, Pamplin WE, Shabsin HS (1982) A double-blind investigation of the relationship between seizure activity and the sleep EEG following EEG biofeedback training. Biofeedback Self-Regul 7(2):193-209

3. Rechtschaffen A, Kales A (1968) A manual of standardized terminology, techniques and scoring system for sleep stages of human subjects. US government printing office, Washington DC

4. Schiff ND, Giacino JT, Kalmar K, Victor JD, Baker K, Gerber M, Fritz B, Eisenberg B, O'Connor J, Kobylarz EJ, Farris S, Machado A, McCagg C, Plum F, Fins JJ, Rezai AR (2007) Behavioural improvements with thalamic stimulation after severe traumatic brain injury. Nature 448(7153):600-603

5. Diner DS (1986) Invasive method of somatosensory evoked potential monitoring. J Clin Neurophysiol 3:113-130

6. Mukhametov LM, Supin AY, Polyakova IG (1977) Interhemispheric asymmetry of the electro-encephalographic sleep patterns in dolphins. Brain Res 134:581-584
7. Mukhametov LM (1984) Sleep in marine mammals. Exp Brain Res 8:227-238

8. Lyamin OI, Mukhametov LM, Siegel JM, Nazarenko EA, Polyyakova IG, Shpak OV (2002) Unihemispheric slow wave sleep and the state of the eyes in a white whale. Behav Brain Res 129:125-129

9. Mukhametov LM (1987) Unihemisphere slow-wave sleep in the Amazon Dolphin, Inia geoffrensis. Neurosci Lett 79:128-132

10. Lyamin OI, Manger PR, Ridgway SH, Mukhametov LM, Siegel JM (2008) Cetacean sleep: an unusual form of mammalian sleep. Neuroscience and Biobehav Rev 32:1451-1484

11. Murayama T, Aoki I, Ishii T (1993) Measurementof the electroencephalogram of the bottlenose dolphin under different light conditions. Aquat Mamm 19.3:171-182

12. Tobayama T, Shimizu H (1973) Relation between food quality and body weight of dolphins in captivity (Maintenance requirement). J Jpn Assoc Zoo Aquarium 37-39

13. Joseph BE, Asper ED, Antrim JE, Pearson JC (1990) Marine mammal transport. In: Dierauf LA (ed) Handbook of marine mammal medicine. CRC, Florida, pp 591-599

14. Veflik AB (1971) Electrical fields and their sampling. In: Kooi KA (ed) Fundamental of electroencephalography. Harper and Row, New York

15. Møhl B, Au WW, Pawloski J, Nachitigall E (1998) Dolphin hearing: relative sensitivity as a function of point of application of a contact sound source in the jaw and head region. Acoust Soc Am 105:3421-3424

16. Nunez PL, Srinivasan R (2006) Electroencephalogram. Scholarpedia 2(2):1348. Available via DIALOG.http://www.scholar pedia.org/article/Electroencephalogram of subordinate document. Accessed 1 March 2006

17. Basmajian JV, DeLuca CJ (1985) Decomposition of the EMG signal and analysis of the motor unit action potential trains. In: Butler J (ed) Muscles alive, 5th edn. Williams and Wilkins, Baltimore 
18. Ohashi J (1993) Effects of contraction level on the changes of surface electromyogram during fatiguing static contractions. Ann Physiol Anthropol 12.4:229-241
19. Morimoto S, Umazume Y, Masuda M (1980) Properties of spike potentials detected by surface electrode in intact human muscle. Jpn J Physiol 30:71-80 